\title{
L'argumentation sur la titulature impériale dans la dynastie Ming au prisme de la "Théorie généralisée de l'argumentation »
}

A Study of the Argumentation about the Imperial Title Issue in Ming Dynasty from the Perspective of Generalized Argumentation Theory

Ju Shi'er, Liu Wen and Chen Zhixi

Translator. Roselyne Koren

\section{(2) OpenEdition} Journals

Electronic version

URL: http://journals.openedition.org/aad/4583

DOI: $10.4000 /$ aad. 4583

ISSN: 1565-8961

\section{Publisher}

Université de Tel-Aviv

Electronic reference

Ju Shi'er, Liu Wen and Chen Zhixi, «L'argumentation sur la titulature impériale dans la dynastie Ming au prisme de la "Théorie généralisée de l'argumentation » », Argumentation et Analyse du Discours [Online], 25 | 2020, Online since 15 October 2020, connection on 17 October 2020. URL : http://journals.openedition.org/aad/4583 ; DOI : https://doi.org/10.4000/aad.4583

This text was automatically generated on 17 October 2020

Argumentation \& analyse du discours est mis à disposition selon les termes de la licence Creative Commons Attribution - Pas d'Utilisation Commerciale - Pas de Modification 4.0 International. 


\title{
L'argumentation sur la titulature impériale dans la dynastie Ming au prisme de la « Théorie généralisée de l'argumentation »
}

\author{
A Study of the Argumentation about the Imperial Title Issue in Ming Dynasty \\ from the Perspective of Generalized Argumentation Theory
}

Ju Shi'er, Liu Wen and Chen Zhixi

Translation : Roselyne Koren

\section{Introduction : « L'argumentation généralisée » et sa méthode de recherche ${ }^{1}$}

1 L'un des traits qui caractérisent l'homme est de raisonner des choses en activant des arguments mis en mots. Par le biais de l'argumentation, il est possible de résoudre des désaccords et d'aboutir à un consensus, en préservant l'harmonie. De nos jours, le développement de l'humanité est entré dans une phase de globalisation qui implique inéluctablement des conflits entre différentes cultures. C'est pourquoi la recherche en argumentation a de plus en plus retenu l'attention. Cette situation appelle en effet une théorie permettant de traiter des différends que provoquent les fossés qui se creusent entre différentes cultures. Nous allons introduire dans cet article la «Théorie généralisée de l'argumentation » et proposer une méthode de recherche permettant de décrire les règles (normes sociales), et les structures de l'argumentation, en partant du point de vue du participant qui prend part à l'activité argumentative. Il s'agit de développer une théorie de l'argumentation favorable au pluralisme culturel et à la diversification - culture dominante incluse (voir également Ju 2020).

2 L'argumentation généralisée est une interaction sociale entre participants dans un certain contexte. C'est, plus précisément, une série d'actes discursifs, dotés d'une 
fonction argumentative, qui sont produits dans un cotexte dynamique par des participants appartenant à une ou plus d'une communauté socio-culturelle ayant ses propres normes (Ju 2010). Afin de découvrir et interpréter les règles et les procédures argumentatives, il nous faut donc dégager les normes sociales ${ }^{2}$ sous-jacentes qui régulent la genèse de l'action discursive.

Dans l'argumentation généralisée, envisagée du point de vue des interactions sociales de la communauté socio-culturelle des participants, l'analyse de la procédure qui génère l'action discursive comprend trois phases : où cela a-t-il lieu (le contexte), que fait cette action (la fonction de l'action discursive) et comment le fait-elle (le mode d'énonciation de l'action discursive). Ceci correspond aux trois questions de recherche à traiter : 1) dans quel contexte l'argumentateur se trouve-t-il lorsqu'il tente une action discursive? 2) quelle fonction l'action discursive remplit-elle dans le contexte en question? 3) à quel discours recourir afin de réaliser cette fonction dans un tel contexte?

Répondre à la première question implique nécessairement de tirer au clair la situation dans laquelle se trouvent les participants lorsque commence l'argumentation; ou de voir à quoi les participants sont confrontés au cours des procédures argumentatives pertinentes, y compris les actes discursifs effectués par d'autres participants. Répondre à la seconde question nécessite que l'on détermine, dans un contexte donné, dans quel genre de normes sociales est ancrée l'action discursive qui permet d'effectuer une argumentation, de sorte que l'action discursive, dans un tel contexte, puisse d'une part être conforme aux normes sociales et d'autre part, avoir une fonction correspondante qui contribue à réaliser l'enjeu argumentatif global. Dans le cadre des théories de l'argumentation, nous qualifions ce type de norme sociale de « règle fonctionnelle de l'argumentation ». Répondre à la troisième question exige que l'on prenne la décision suivante : lorsque le participant a décidé de la fonction à attribuer à l'action discursive, dans un contexte donné, conformément à la règle fonctionnelle, il/elle aura à décider quel discours spécifique choisir pour accomplir l'action discursive et mettre en œuvre la fonction prévue. Du fait que l'action discursive effectuée dans la procédure argumentative est d'ordre social, le discours sélectionné doit également être conforme aux normes sociales. Nous qualifions de "règles énonciatives de l'argumentation » les normes sociales auxquelles le discours doit se conformer.

5 Sur la base des règles énoncées ci-dessus, l'argumentation généralisée peut être décrite comme un système d'interaction sociale dans un contexte dynamique.

1. Des membres de la société sont en désaccord, dans un contexte donné, et sont prêts à élaborer des discours dotés de fonctions argumentatives. C'est ainsi que s'enclenche l'argumentation.

2. Un participant (locuteur) détermine, en fonction du contexte où il/elle se trouve, le rôle que l'action discursive va jouer conformément à la règle fonctionnelle.

3. Un locuteur configure un discours apte à réaliser la fonction ci-dessus conformément à la règle énonciative, et à effectuer ensuite l'action discursive.

4. L'auditeur comprend l'action discursive et d'autres effets qui s'ensuivent, et modifie le contexte d'origine. Puis l'auditeur change de rôle et devient à son tour locuteur, d'où un retour à 2 , dans le nouveau contexte.

... (et ainsi de suite)

6 L'argumentation prend fin lorsque la visée argumentative est atteinte ou que les participants y mettent fin. 
7 La visée de la recherche dans la Théorie généralisée de l'argumentation consiste à fournir une méthode permettant de dégager les règles en question et de décrire la procédure dynamique de l'argumentation généralisée. Étant donné que l'argumentation, énoncée en langue naturelle dans une communauté socio-culturelle particulière, est conforme aux normes sociales de la communauté, cette méthode consiste à respecter le principe de localisation : elle se refuse à décrire l'argumentation d'une culture dans le cadre conceptuel d'une autre culture. Elle mettra l'accent sur les normes de la communauté socio-culturelle à laquelle les participants appartiennent et décrira les règles et systèmes de l'argumentation du groupe dans un contexte dynamique. Par conséquent, la procédure localisée d'une argumentation généralisée peut être brièvement représentée comme suit (Ju 2019) :

1. Réunir les informations socio-culturelles contextuelles pertinentes;

2. réunir les données empiriques de l'argumentation via l'investigation qualitative du champ de recherche ;

3. analyser les données de 2 sur la base de 1 et trouver les règles argumentatives et les stratégies en jeu grâce à l'explication des actions discursives argumentatives concernées ;

4. justifier, en fonction de 1 , la raisonnabilité des règles et des stratégies de l'argumentation sélectionnée ;

5. contrôler les règles de l'argumentation justifiées en 4 dans leur contexte.

8 Parmi elles, l'enquête qualitative dite de terrain (stade 2) renvoie à l'exploration méticuleuse des paroles sociales de l'Autre effectuée à travers une interaction longue, complète et authentique avec les autres là où ils résident; cette interaction récolte, autant que possible, des matériaux de première main concernant leur environnement, leurs difficultés, origines, langage, rituels et rapports sociaux afin de mieux pouvoir les comprendre (Van Maanen $2011: 1-3$ ). Toutefois, afin de comprendre les autres, il est nécessaire de comprendre leur histoire. Il est également nécessaire de pratiquer une investigation dite de terrain: de se procurer les textes sur «l'environnement, les difficultés, les origines, le langage, les rituels et rapports sociaux » des autres au long de leur histoire. Ce n'est pas uniquement pour apporter des preuves en citant la littérature historique : c'est pour faire l'expérience de la vie des autres et y pénétrer - comme dans le cas des Azandé (cf. Ju 2010). Nous pouvons étudier une argumentation historique (Ju et He 2014) en nous fondant sur l'investigation sur le terrain du texte qualitatif.

9 Les sciences empiriques ont pour but de décrire les lois universelles du monde empirique et leurs méthodes partagent toutes les procédures suivantes: (1) comprendre les théories scientifiques: les termes scientifiques, les hypothèses fondamentales, les lois, l'observation et les méthodes expérimentales scientifiques; (2) recueillir des données empiriques; (3) accéder à une hypothèse par induction ou instinct ; (4) effectuer l' analyse théorique de l'hypothèse ; (5) vérifier l'hypothèse par des expériences. Les méthodes des sciences empiriques et celles de la Théorie de l'argumentation généralisée partagent des traits similaires dans la mesure où toutes deux sont ancrées dans l'observation et l'analyse des données. Il y a aussi toutefois quelques différences significatives. Les sciences empiriques sont à la recherche de régularités dans des domaines empiriques par induction et statistiques, tandis que la Théorie de l'argumentation généralisée a pour fin d'explorer, par l'intermédiaire de l'explication et de la justification d'actions, les normes sociales auxquelles les 
argumentateurs d'une communauté socio-culturelle particulière doivent se conformer lorsqu'ils débattent verbalement les uns avec les autres.

Nous allons avoir recours, dans le reste de cet article, à la procédure localisée de la Théorie généralisée de l'argumentation afin d'étudier la question de la titulature impériale dans la Controverse du Grand Rituel de la dynastie Ming. Nous allons présenter, dans la section 2, les origines culturelles de la Controverse du Grand Rituel. Nous allons introduire en particulier l'éthique et les institutions - telles la loi du clan et les traditions de la piété filiale, les rouages gouvernementaux, etc. La section 3 est consacrée à la question du titre impérial, l'une des 7 questions de la Controverse du Grand Rituel. Nous allons évoquer l'origine, les principaux participants au débat sur la question et les principaux rouages de la Controverse du Grand Rituel. Nous allons nous livrer, dans la section 4, étape par étape, à une analyse de la question du titre impérial et découvrir les règles argumentatives employées par les participants dans le déroulement progressif de l'affaire. Après l'analyse, l'interprétation socio-culturelle aidant, nous dégagerons les règles et le système de l'argumentation sur l'affaire en question dans le cadre de la Théorie Générale de l'Argumentation. Puis nous vérifierons la validité des règles indiquées plus haut.

\section{Origines culturelles de la Controverse du Grand Rituel}

\section{1. La tradition de la loi du clan}

11 La société chinoise traditionnelle avait la loi du clan pour fondement naturel (Liu 2011: 1). La loi du clan a été instaurée et pratiquée par la dynastie Zhou (1046-256 av.- J.-C.), avec, en son centre, le principe de primogéniture. «La loi du clan (Zong Fa Zhi) réfère à l'origine à l'éthique du système féodal qui a les liens du sang pour fondement, et qui est institutionnalisé sur la base de la supériorité du statut social et de l'ancienneté d'âge, système qui prend le patriarcat pour principe et les progéniteurs (grands ou mineurs) pour standard» (Li 1988: 54). Selon le Han Yu Da Ci Dian (Le Grand dictionnaire chinois):

Tous les fils aînés légitimes ${ }^{3}$ de la lignée établie par le sang sont de grands progéniteurs et les fils cadets des progéniteurs mineurs. Le trône impérial doit être attribué au fils aîné légitime qui doit être qualifié de grand progéniteur. Tous les autres fils sont des progéniteurs mineurs par rapport au grand progéniteur. Toutefois, dans leurs propres fiefs territoriaux, les princes transmettent aussi leurs trônes, en tant que chefs, aux fils aînés légitimes qui sont par rapport aux autres fils, les progéniteurs mineurs, de grands progéniteurs.

Parfois, lorsque meurt le fils aîné légitime, l'aîné de la branche légitime des petits-fils monte sur le trône. Lorsque le fils aîné légitime n'a pas d'héritier, son jeune frère consanguin sera couronné selon un ordre de succession qui repose sur l'ancienneté de l'âge. C'est ce qui est consigné dans Chun Qiu Gong Yang Zhuan en ces termes: " la légitimité d'un héritier du trône a l'ancienneté de l'âge pour fondement plutôt que le jugement qui évalue s'il est ou non vertueux ». S'il n'y a pas de fils légitime pour monter sur le trône, la légitimité revient à d'autres fils, issus des deuxièmes épouses de l'empereur. (Qu 2017 : 110). 


\section{2. la tradition de la piété filiale}

13 La piété filiale est la norme éthique selon laquelle «bien traiter ses parents » est fondamental. Dans la dynastie Zhou de l'ouest (1046-771 av. J.-C.), une époque antérieure à l'ère de Confucius, la piété filiale était déjà une notion éthique universelle. Confucius a instauré un système fondé sur la bienveillance, considérant celle qu'on manifeste à ses parents et ses frères comme le socle et la prémisse de la bienveillance en général, et de ce fait, il a enrichi la signification de la piété filiale. L'ouvrage Xiao Jing (un classique de la piété filiale) rédigé au début de la dynastie Han (202 av.-J.C- 220 apr.J. C.) a étendu le principe de piété filiale du fait de «bien traiter ses parents » à celui de « diriger le pays au nom de la pitié filiale » (Zhang 2000 : 77).

La piété filiale joue un rôle extrêmement important dans la vie traditionnelle quotidienne des Chinois comme dans la politique. Elle est

le fondement spirituel et le point de départ pratique de toutes les interactions verbales dans la société chinoise. Elle est aussi le fondement éthique et spirituel de la politique chinoise du passé. Elle occupe une place centrale dans l'endoctrinement social et l'éducation scolaire, et a un impact considérable sur la façon dont les Chinois s'habillent, mangent, se logent, voyagent, sur leur mode de vie, le folklore et les arts (Xiao $2000: 33$ ).

\subsection{Les empereurs Ming, le cabinet intérieur et le Ministère des Rites}

La dynastie Ming (1368-1644) est une monarchie et l'empereur jouit du pouvoir suprême dans la hiérarchie du pouvoir. Il y a deux façons essentielles de traiter les affaires politiques de l'État. La première consiste à faire appel à des officiels pour discuter d'affaires politiques. La seconde consiste à promulguer des décrets, à lire des mémorandums et à y répondre. Les officiels se servent essentiellement de divers mémorandums pour signaler ou présenter leurs idées aux empereurs, mémorandums qui doivent être lus par ces derniers, donner lieu à des réponses, puis être transférés aux ministères concernés pour exécution. (Zhang et Lin 1528 : 201, 205-206).

Zhu Yuanzhang (1328-1398)4, le fondateur de la dynastie Ming, a aboli le Secrétariat et le système des conseillers; il a redistribué leurs responsabilités à six ministres et attribué la fonction de conseiller à un groupe d'officiels érudits, qui est ultérieurement devenu le cabinet intérieur. Par la suite, le cabinet intérieur s'est encore amélioré et ses grands secrétaires ont assumé une part des responsabilités assumées auparavant par les conseillers du Secrétariat ${ }^{5}$. Le nombre des grands secrétaires variait entre un et plusieurs membres, parmi lesquels le responsable de l'ensemble du groupe était le grand secrétaire en chef. Comme le cabinet intérieur est plus proche des empereurs et communique davantage avec eux, il exerce l'influence la plus forte sur les affaires politiques de la dynastie Ming (Wang $2014: 48$ ).

Le Ministère des Rites, l'un des six ministères, est responsable des rituels de l'État, des rituels sacrificiels, de la vie diplomatique et des banquets de l'État, etc.; ceux qui sont à leur tête ont le titre de ministre. Conformément au système juridique de la dynastie Ming, lorsque l'empereur montait sur le trône, sa mère était en principe élevée au rang d'impératrice et devait recevoir un titre honorifique. Un rituel devait par la suite faire honneur à l'impératrice fraîchement nommée, deux ou quatre caractères honorifiques étaient ajoutés à son titre (Zhang $2014: 1745)$. 


\subsection{Huang Ming Zu Xun (Injonctions ancestrales)}

(lois ancestrales) est un énoncé extrêmement fréquent, ce qui indique son importance dans les activités politiques. Zu Zhi réfère, littéralement, au système de lois codifiées, compilées par le fondateur de la dynastie Ming lui-même, Zhu Yuanzhang, système dans lequel Huang Ming $\mathrm{Zu}$ Xun bénéficie de la plus grande déférence et autorité $\mathrm{Wu}$ 1991 : 27).

Le Huang Ming Zu Xun comprend 13 catégories en termes de contenu. Zhu Yuanzhang déclara avec emphase, afin que ses descendants s'y conforment, qu' « il ne saurait être question d'en modifier le moindre mot " (Zhu 1996: 172). Le Huang Ming Zu Xun fut considéré comme un ensemble de « lois divines » par les empereurs et les officiels. La plupart des injonctions qu'il contient peuvent être suivies par les descendants. Toutefois, lorsque l'empereur ne le respecte pas, les officiels veilleront à le lui reprocher. Et même si leur reproche n'a pas d'effet, il peut être leur talisman (Wu 1991 : 27).

\section{La Controverse du Grand rituel : son origine, ses solutions, ses procédures}

\subsection{Son origine}

Le 20 avril 1521, Zhu Houzhao (1491-1521), le dixième empereur de la dynastie Ming meurt de maladie à l'âge de 31 ans, le temple honorifique auquel on donne le nom d'« Empereur Wuzong " lui est dédié à titre posthume. Comme il n'a pas de descendant ni de frères de sang, il ne laisse personne habilité à accéder légitimement au trône, ce qui suscite la crainte d'une déstabilisation de l'État. Après s'être assuré du soutien de la mère de Wuzong, l'impératrice Cishou (1470-1541 A. D.) et des eunuques de la cour, Yang Tinghe (1459-1529), alors grand secrétaire en chef, publie et rédige l'Édit de succession de l'empereur Wuzong au nom du défunt empereur, où on peut lire :

\footnotetext{
Je me meurs à présent, mais je n'ai pas nommé d'héritier potentiel. Zhu Houcong, le fils aîné du plus jeune frère consanguin de mon père, le prince Xian de Xing, est à présent adulte. Il est vertueux, talentueux, bienveillant et se conduit bien avec ses parents. Il doit être couronné empereur conformément à la loi de l'ordre régulant la succession. Respectant en cela l'injonction quand-le-frère-le-plus-âgé-meurtcouronnez-le-plus-jeune-frère, le moment est venu de le déclarer au temple ancestral de la famille et d'en informer l'impératrice Cishou afin que les officiels soient immédiatement envoyés pour aller le chercher et l'amener à Pékin, afin qu'ils le couronnent empereur et effectuent le rituel sacrificiel dans le temple de sorte qu'il puisse diriger l'État (Yang Tinghe, Fei Hong et al., vol. 197 : 4-5)
}

Afin d'agir conformément à l'instruction ancestrale selon laquelle « quand il n'y a pas d'héritier légitime au trône, au moment de la mort de l'empereur ", la légitimité de la succession passe au frère cadet consanguin de l'empereur, Yang Tinghe est remonté d'une génération dans la lignée impériale afin de trouver le successeur légitime : Zhu Youyuan le prince Xian de Xing (1476-1519) est Wuzong, père de l'empereur Xiaozong, le frère cadet consanguin de l'empereur (1470-1505). A l'époque, Zhu Youyuan était déjà décédé, mais il avait un fils unique, Zhu Houcong (à savoir l'empereur Jiajing 
1507-1521-1567) et ce fils fut nommé successeur au trône. Telle est la loi de l'ordre de succession mentionnée dans l'édit.

Le 30 mai 1521, trois jours après l'ascension ${ }^{6}$, Zhu Houcong, le nouvel empereur âgé de quinze ans, envoya des personnes à $\mathrm{An} \mathrm{Lu}$, Hubei pour amener sa mère Jiang la princesse Xian de Xing (?-1538) à Pékin. Le cinquième jour après l'ascension, il envoya les représentants du Ministère des Rites discuter des rites et du titre honorifique destinés à son défunt père (Zhang 1962-68 : 24). Les rites et le titre à discuter avaient pour enjeu la question de savoir comment honorer son père et quel titre lui attribuer : les solutions retenues détermineraient aussi le titre et le statut de sa mère qui était encore en vie (Tian 2013 : 49). Le nouvel empereur le fit, guidé par son amour filial et par piété filiale à l'égard de ses parents, de la sorte que sa demande parut raisonnable. Toutefois, il était une progéniture mineure et avait "accédé au trône par une “branche" collatérale» (Yang 1528: 10b-11a). Ce n'était pas conforme au principe de succession quand-le-frère-aîné-meurt-couronnez-le frère-cadet, qui exige que le successeur soit un frère cadet consanguin. Donc les officiels, sous la férule de Yang Tinghe, insistèrent sur le fait que le nouvel empereur devait auparavant se déclarer descendant (et également enfant adoptif) de son prédécesseur l'empereur Xiaozong et lui succéder seulement alors sur le trône impérial. Le nouvel empereur insista cependant sur sa succession au trône, mais pas sur l'injonction de la descendance, donnant ainsi le coup d'envoi de la Controverse du Grand Rituel (Zhao 2012 : 9).

\subsection{Deux solutions ${ }^{7}$}

23 Les officiels proposèrent, dans le feu de la discussion, trois solutions concernant le titre honorifique devant obligatoirement être attribué au père de l'empereur Jiajing.

\subsubsection{Le « descendantiste »}

24 La première solution fut proposée par la majorité des officiels avec pour porte-parole le grand secrétaire en chef Yang Tinghe et le ministre des rites Mao Cheng. Dans le but d'éviter que la lignée de l'empereur Xiaozong à l'empereur Wuzong ne soit rompue, ils proposèrent que la succession par adoption précède la succession de la ligne dynastique ; aussi insistèrent-ils pour que l'empereur Jiajing appelle son prédécesseur l'empereur père et Zang (la femme de Xiaozong) l'impératrice mère et qu'il appelle son père biologique "oncle " et sa mère biologique "tante ", ce qui revenait à renier sa parenté biologique. Cette solution proposait aussi, en outre, que son cousin, le second fils de l'oncle de Jiajing, le prince de Yi, prenne, en tant que fils, la place de Jiajing et vive sur les terres de son père de sorte que les rites sacrificiels dédiés au père biologique de Jiajing puissent être maintenus. Le ministre des rites prépara les titres honorifiques "oncle de l'empereur le Grand prince Xian de Xing" et "tante de l'empereur la princesse Xian de Xing» pour les parents biologiques du nouvel empereur et ce dernier devait se servir de "neveu empereur » en présence de ses parents biologiques ou dans sa correspondance avec eux (Yang 1528 : 9b-12a). Comme la majorité des officiels soutenait cette solution, les chercheurs les qualifient de Parti de la majorité, ou de Parti descendantiste. Nous les appelons, dans cet article, pour faire court, « les Descendantistes » (voir la proposition des Descendantistes ci-dessous) 


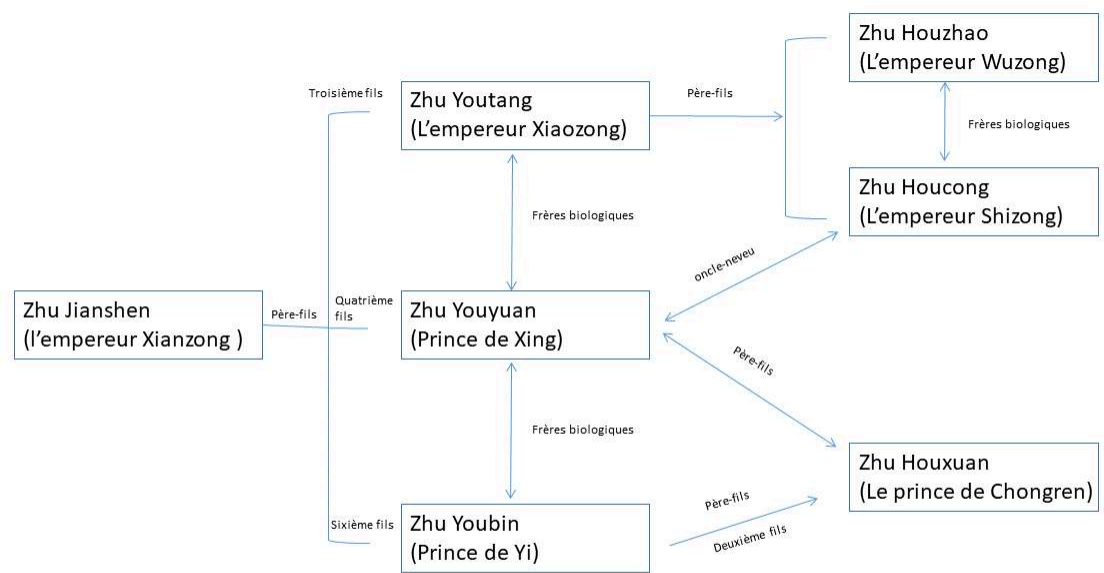

Tableau 1. La proposition descendantiste (Tian $2013: 53$ )

\subsubsection{L'humaniste}

La seconde solution fut proposée par Zhang Cong et soutenue par plusieurs officiels au statut inférieur-moyen. Ils firent l'éloge de l'amour filial et de la piété dont le nouvel empereur avait fait preuve afin d'honorer ses parents biologiques. Ils pensaient que le fondement des rites était l'humanité et que supprimer les liens naturels de l'empereur avec ses parents était une erreur. Zhang Cong proposa de séparer la succession par adoption de la succession par appartenance à la lignée dynastique et que le nouvel empereur accède au trône par la lignée dynastique de Xiazong à Wuzong, mais ne modifie pas sa relation à ses parents biologiques ni sa relation au précédent empereur (Zhang 1962-68 : 160-p ; 1528 : 5b-17a). Comme cette solution n'était agréée que par un petit nombre de partisans, les chercheurs les qualifièrent de Parti de la minorité ou de Parti de la Succession-dans-la Lignée-dynastique. Nous allons les nommer, dans cet article, les "Humanistes", parce que leurs propositions ont des considérations humaines pour fondement (voir ci-dessous la proposition des Humanistes) 


\subsection{La procédure}

La Controverse du Grand Rituel a pour but de résoudre le problème des rites et des titres honorifiques du prince Xian de Xing ; la résolution du premier problème sert de fondement à celle du second. Confucius dit dans les Entretiens-Zi Lu que «si les noms ne sont pas rectifiés, le langage ne fonctionnera pas correctement, et si le langage ne fonctionne pas correctement, ce que l'on entreprend ne réussira pas» (Watson 2007). Donner un titre honorifique, c'est corriger les noms.

D’après le résumé de Zhang Cong dans Ming Lun Da Dian (voir ci-dessous), compilé en 1528, plus de 700 officiels ont participé aux discussions et il y eut plus de 300 mémorandums soumis au tribunal. Le tribunal fut pris dans son ensemble dans une controverse de longue durée et à vaste échelle, portant sur sept questions (Yang 1528 Vol. $24:$ 10). Parmi elles, les trois premières résolvent le problème du titre honorifique et les quatre autres celui des rites ${ }^{8}$.

La première question est celle de la paternité : le débat pour savoir qui Jiajing serait obligé de considérer comme son père (ce qui s'applique aussi à sa mère). C'est la question fondamentale dans la Controverse du Grand Rituel. La question du titre d'empereur est la seconde et c'est un débat qui porte sur le fait d'honorer le père de l'empereur, le prince Xian de Xing en le qualifiant d'empereur Xingxian, ce dont nous allons bientôt discuter en détails. Jiajing voulut, après la question du titre impérial, faire davantage encore honneur à ses parents, et cela fut à l'origine de la question du titre de majesté. En mai 1525, un officiel suggéra que la tablette spirituelle de l'empereur Xingxian soit placée dans le temple royal ancestral et ouvrit ainsi la voie à la question du temple. En août 1526 un temple spécialement dédié au père de Jiajing fut 
inauguré, et cela eut pour conséquence de soulever quelques questions concernant la construction de la route menant à ce temple, l'éventualité que la mère de Jiajing puisse entrer dans le temple pour une offrande sacrificielle et les sortes de musique et de danse, par courtoisie, appropriées au temple - d'où la question de la route, celle de la visite au temple et celle de la musique et de la danse.

Voyant que tous ses buts avaient été atteints, lors des débats, l'empereur Jiajing convoqua les officiels afin qu'ils compilent un livre rapportant les propos et actes du ministre, donnant ainsi un résumé officiel de la Controverse du Grand Rituel. Le livre fut achevé un an et demi plus tard et l'empereur l'intitula Ming Lun Da Dian (La grande cérémonie Minglun). La controverse qui avait duré des années prit fin à cette époque-là 9 .

\section{L'argumentation dans l'affaire du titre impérial}

Parmi les sept questions, celle du titre impérial a été la première conclue. Notre analyse se concentre sur cette question particulière. En suivant les différences de temps et de contenu dans cette affaire, on se focalisera sur sept actions discursives. Pour la commodité de la lecture, on numérotera les actions au début des paragraphes qui en traitent. Qui plus est, les participants de la controverse seront représentés comme suit :

Le Descendantiste : Les grands secrétaires $\left(\mathrm{Z}_{1}\right)^{10}$, les officiels du Ministère des Rites

$\left(\mathrm{Z}_{2}\right)^{11}$

L'Humaniste : L'empereur Jiajing $\left(\mathrm{R}_{1}\right)$, Zhang Cong $\left(\mathrm{R}_{2)}\right.$

\section{1. L'affaire du titre impérial}

\subsubsection{La proposition du descendantiste}

31 Comme d'ores et déjà évoqué dans la section 3.1., après la succession au trône, l'empereur Jiajing $\left(\mathrm{R}_{1}\right)$ chargea le ministère des rites de discuter des rites appropriés et $\mathrm{du}$ titre honorifique de son défunt père. Le ministère des rites était responsable, conformément au système politique Ming, des rites sacrificiels et de quelques autres encore. Dès la réception des ordres des empereurs, il devait intimer aux officiels de débattre et de faire des propositions, puis de présenter les résultats de la discussion aux empereurs. Mao Cheng (1461-1523), ministre des rites, alla consulter le grand secrétaire en chef Yang Tinghe et convoqua des officiels afin de débattre avec eux. Ils déduisirent de la discussion que dans deux événements historiques similaires concernant l'empereur Ai (25-7-1 av. J.-C.) de la dynastie Han et l'empereur Ying (1032-1063-1067) de la dynastie Song, on avait recouru au principe de la loi du clan selon lequel « une personne descendante d'une autre personne est le (la) fils/fille de cette dernière ». Donc également soutenu par la signature de 69 autres officiels, $\left(\mathrm{Z}_{2}\right)$ présenta le résultat du débat à l'empereur dans un mémorandum signé où figurait la proposition suivante :

(1) Avant de monter sur le trône Ai empereur de Han et Ying empereur de Song étaient des progéniteurs mineurs. Étant donné que les deux empereurs précédents n'avaient pas d'héritiers légitimes, ils furent déclarés descendants de leurs prédécesseurs et devinrent de grands géniteurs. Ils voulurent, tous deux, élever le statut de leurs pères et leur attribuer des titres honorifiques, ce qui provoqua des débats animés et une forte opposition. Sima Guang (1019-1086), un grand érudit de Song, et Cheng Yi (1033-1107) 
un grand penseur de Song, pensaient que les descendants devaient être les fils de ceux dont ils descendaient et que le fait d'être devenu un grand progéniteur tout en étant, à l'origine, un progéniteur mineur, devrait les inciter à mettre un terme aux tentatives d'honorer leurs parents biologiques. D'où la conclusion des Descendantistes: l'empereur Jiajing est dans l'obligation d'appeler père l'empereur Xiaozong, et de nommer son père biologique, le prince Xiang de Xing, oncle de l'empereur, l'impératrice Zhang (femme de Xiaozong), mère et sa propre mère biologique, la princesse Xian de Xing, tante de l'empereur (voir la section 3.2.1. - Yang 1528 : Vol.2 : 9b-12a).

On retiendra de l'analyse ci-dessus que l'action discursive (1) a suivi les règles argumentatives suivantes. Tout d'abord, la règle fonctionnelle de (1) : dans le cas du contexte où l'empereur demande au Ministère des Rites de discuter les rituels afin d'honorer ses parents, le ministère, ayant pour fondement le principe de la loi du clan stipulant qu' " une personne descendante d'une autre personne est un fils ou une fille de cette dernière ", pouvait choisir de proposer à l'empereur qu'il prenne la succession $\mathrm{du}$ grand progéniteur et nomme son père et sa mère biologiques oncle et tante. La règle énonciative de (1) est: dans le cas du contexte où l'empereur demande au Ministère des Rites de discuter les rituels afin d'honorer ses parents, le ministère peut choisir de présenter un mémorandum se référant à des événements historiques antérieurs et réaliser la fonction de faire une proposition.

\subsubsection{La proposition de l'Humaniste}

Après avoir reçu le mémorandum du Ministère des Rites, l'empereur Jiajing répondit à la proposition avec mécontentement et posa la question : " quelqu'un peut-il modifier ses liens parentaux ? » Puis il donna l'ordre qu'on lui fasse une autre proposition (Yang 1528, Vol. 2 : 12a). Par la suite le Ministère des Rites eut deux autres conventions afin de mener des débats supplémentaires et présenta ses mémorandums à l'empereur. Le cabinet intérieur, dirigé par le grand secrétaire en chef Yang Tinghe, fit simultanément deux tentatives et présenta ses mémorandums à l'empereur. Toutes leurs propositions concernant cette question avaient la loi du clan pour fondement et ne furent pas acceptées par l'empereur Jiajing. Il n'avait toutefois que quinze ans, ne savait pas grand-chose sur les rites et n'était pas capable de fournir de bons arguments pour les blâmer. Donc, il décida d'attendre.

Selon les lois ancestrales de la dynastie Ming, n'importe qui pouvait rendre compte de situations aux empereurs et exprimer des opinions devant eux. En août 1521, Zhang Cong, un étudiant du Ministère des Rites, présenta un mémorandum $\mathrm{Da}$ Li Shu à l'empereur, dans lequel il proposa une idée humaniste s'inspirant d'une idée du classique confucéen $\mathrm{Li} J \mathrm{~J}$ selon lequel «les rites concernent les relations humaines naturelles", mémorandum qui était en contradiction avec la proposition du Descendantiste. L'empereur Jiangjing considéra que c'était là une bonne occasion. Cet article comprenait la démarche de principe à suivre pour les idées humanistes, incluant ce qui suit :

(2) Premièrement, il prenait acte de la décision du nouvel empereur d'honorer son père biologique puisque cette décision manifestait l'amour et la piété filiaux de l'empereur, ce qui correspond à une valeur reconnue par l'État: gouverner au nom de la piété filiale. Deuxièmement, une citation du Li Ji fournit une raison d'être à leur argument : «Le rite ne provient ni du ciel ni de la terre. Il ne concerne que les relations 
humaines ". Troisièmement, les deux cas historiques similaires ne peuvent pas servir de base à la controverse du grand rituel parce que et l'empereur Ai de Han et l'empereur Ying de Song s'étaient occupés de l'enfant adopté au palais, montrant ainsi apparemment leur intention de faire des adoptés leurs descendants et successeurs. Toutefois, dans le cas de l'empereur Jiajing, l'empereur Wuzong, cousin de Jiajing, ne fit jamais aucune démarche en vue d'une adoption. Donc, du point de vue de la considération de la relation humaine, et des rites dûs au père de l'empereur Jiajing, l'empereur doit garder ses parents biologiques pour parents et ériger un temple pour son père à Pékin. La princesse Xian de Xing, sa mère biologique, doit bénéficier simultanément de cet honneur au même titre que lui (Yang 1528, vol. $3: 5 \mathrm{~b}-17 \mathrm{a}:$ Zhang 1962-68, vol. $4: 160$ ).

37 On déduit de l'analyse ci-dessus que l'action discursive (2) comprend les règles argumentatives suivantes. La règle fonctionnelle de (2) est tout d'abord: dans le contexte où l'empereur veut honorer ses parents et où le Ministère des Rites a fait une proposition concernant la succession et le changement de nomination, les sujets, personnalités officielles ou simples mortels, sont autorisés à faire à l'empereur des suggestions sur la façon d'honorer ses parents, fondées sur le principe que les rites concernent les relations humaines dans la nature. Deuxièmement, la règle énonciative (2) est : dans un contexte où l'Empereur veut honorer ses parents et où le Ministère des Rites a fait une suggestion sur la succession et le changement de nomination, les sujets pourraient choisir de citer les classiques confucéens pour offrir des conseils à l'empereur.

\subsubsection{L'ordre de l'empereur Jiajing}

Conformément au système politique de la dynastie Ming, les grands secrétaires servaient de conseillers à l'empereur et ils discutaient fréquemment d'affaires importantes de l'État avec les empereurs. Dès que ces derniers avaient pris une décision, ils publiaient des décrets afin qu'elle soit exécutée. Après la soumission du mémorandum de Zhang Cong, l'empereur l'envoya, après l'avoir lu, aux secrétaires et déclara : «Les points soulevés dans ce mémorandum correspondent bien au Huang Ming $\mathrm{Zu}$ Xun et sont fondés sur d'anciens rites. Pourquoi me maltraiter de la sorte (et me demander de modifier ma parenté) ? » Yang Tinghe répondit : « Zhang Cong n'est qu'un étudiant et il ne sait rien des affaires de l'État». Un nouveau mémorandum de Zhang Cong fut à nouveau soumis à l'empereur. Après l'avoir lu attentivement, l'empereur dit avec joie: "Selon cette opinion, ma relation avec mon père peut finalement rester intacte » (Yang 1528, vol. 3 : 12a). Puisque la piété filiale était le fondement de l'éthique politique de la dynastie Ming, l'empereur décida de persuader les grands secrétaires en faisant appel aux principes des relations humaines et de la piété filiale et donna l'ordre suivant :

(3) Vers la fin août, l'empereur a convoqué les trois grands secrétaires et les a rencontrés au palais Wenhua. Il leur a offert du thé et leur a dit : «Mes parents sont les personnes que j'aime le plus. Je vous prie de comprendre mon profond amour pour eux. » Puis il leur donna un décret écrit stipulant que «même si ce que vous avez dit était raisonnable, mes parents ont été si bons avec moi que je n'ai pas encore pu leur rendre la pareille pour leurs efforts consacrés à mon éducation. Accordez à présent à mon père le titre d'empereur Xingxian et à ma mère celui d'impératrice Xingxian » et il leur demanda d'agir en fonction (Yang 1528, vol. 4 :1a-2b). 
Nous voyons, grâce à la mise au point ci-dessus, que l'action discursive (3) comprend les règles argumentatives suivantes: la règle fonctionnelle de (3) est, tout d'abord: dans le contexte où les personnalités officielles ne sont pas d'accord avec l'empereur quant à l'acte d'honorer ses parents et où Zhang Cong a fait une proposition soutenant l'empereur, l'empereur pouvait choisir de maintenir sa décision d'honorer ses parents en fonction du critère de la piété filiale selon lequel la progéniture doit récompenser ses parents. La règle énonciative de (3) est, deuxièmement, la suivante: dans un contexte où les officiels ne sont pas d'accord avec l'empereur concernant l'honneur dû à ses parents et où Zhang Cong a fait une proposition afin de soutenir l'empereur, l'empereur peut choisir de rédiger un décret invoquant la piété filiale et les relations familiales pour activer la fonction ci-dessus

\subsubsection{Rejet de l'ordre}

41 Conformément à la fonction du cabinet intérieur, les grands secrétaires peuvent donner des conseils aux empereurs au sujet de ce qu'ils ont dit ou fait, ce qui signifie que s'ils pensent que les empereurs ont agi de façon inappropriée ou violé des lois ancestrales, ils peuvent même rejeter ses ordres. Ainsi, après leur réunion avec l'empereur et après avoir reçu son décret, Yang Tinghe et les autres étaient toujours en désaccord avec lui et lui soumirent un autre mémorandum, exprimant les idées suivantes s'inspirant des deux empereurs Shun et Yu, renommés dans l'histoire ancienne pour leur sagesse :

(4) Ce n'est pas qu'ils n'ont pas compris l'empereur, mais ils doivent respecter la tradition: "une personne descendante d'une autre est un fils/une fille pour cette dernière » - formulée dans Chun Qiu Gong Yang Zhuan. Être des descendants signifie que l'on se résigne à ignorer les relations familiales originelles. En s'inspirant davantage encore d'anciens exemples de l'empereur Shun et de l'empereur Yu, on peut voir que tous deux étaient des souverains et qu'ils n'honorèrent pas leurs pères respectifs, Gusou et Gun, après être montés sur le trône. Était-il possible de les blâmer pour ne pas avoir respecté la piété filiale due aux parents? L'empereur de Song Yingzong avait l'intention d'honorer son père biologique, le prince de $\mathrm{Pu}$, mais fut obligé de céder aux officiels en raison de leur désapprobation. On formula l'espoir que l'empereur puisse s'inspirer des empereurs Shun et Yu et traiter ce cas correctement, du moins mieux que l'empereur Yingzong. Cette question était de l'ordre du rituel de l'État et il fallait la traiter sérieusement. C'est la raison pour laquelle ils insistèrent pour que l'empereur Jiajing fasse ce que le Ministère des Rites avait proposé et ils renvoyèrent le décret à l'empereur.

Par conséquent l'action discursive (4) comprend les règles argumentatives suivantes. Premièrement, la règle fonctionnelle de (4) est : dans un contexte où l'empereur convoque des personnalités officielles à des réunions et où ordre leur a été donné par l'empereur d'honorer ses parents, les officiels peuvent éventuellement choisir de rejeter cet ordre, conformément au principe de la loi du clan selon lequel «une personne étant la descendante d'une autre personne est un fils/une fille pour cette dernière ». Deuxièmement, la règle énonciative de (4) est: dans un contexte où l'empereur a convoqué des personnalités officielles à des réunions et où un ordre leur a été donné par l'empereur d'honorer ses parents, les officiels peuvent éventuellement recourir à l'évocation d'événements historiques pour réaliser la fonction ci-dessus. 


\subsubsection{La menace d'abdication}

44 officiels insistant sur les lois du clan versus l'empereur maintenant son soutien au principe de piété filiale. Conformément à ce dernier, la progéniture doit veiller au bien de ses parents. Lorsque la progéniture travaillait ailleurs, elle devait toujours continuer à s'occuper de ses parents. Donc, peu après être monté sur le trône, l'empereur Jiangjing envoya des officiels amener sa mère biologique Jiang $\left(\mathrm{R}_{3}\right)$ à Pékin. Lorsqu'elle arriva à Tongzhou, un faubourg de Pékin, elle entendit parler de la discussion sur les rites et des propositions des officiels. La proposition que son fils unique prenne l'empereur Xiaozong pour père et l'impératrice Cishou (l'épouse de Xiaozong) pour mère l'irrita et elle dit avec colère : «Comment est-il possible d'obliger mon fils à être le fils d'une autre personne? ». Elle refusa d'entrer dans Pékin et resta à Tongzhou (Gu 2015). Quand il vit que sa mère refusait d'entrer dans Pékin à cause de la question du rituel, l'empereur fut extrêmement contrarié et il dit, en gémissant, à l'impératrice Cishou:

(5) « je veux abdiquer et retourner à la maison avec ma mère » (Yang 1528, vol. 4 : 19b). eut donc déduire de ce développement que l'action discursive (5) suit les règles suivantes. Premièrement, la règle fonctionnelle de (5) est: dans un contexte où des officiels ne sont pas d'accord avec un empereur de la dynastie Ming et où l'empereur ne peut pas rencontrer sa mère, l'empereur peut éventuellement choisir la fonction des menaces, conformément au principe de la piété filiale, et veiller au bien de ses parents. Deuxièmement, la règle énonciative de (5) est : dans un contexte où des officiels sont en désaccord avec un empereur de la dynastie Ming et où l'empereur ne peut pas rencontrer sa mère, l'empereur peut choisir d'abdiquer afin de réaliser la fonction cidessus.

\subsubsection{Da Li Huo Wen}

À partir du moment où l'empereur déclara sa volonté d'abdiquer, les officiels prirent peur. Personne n'osa cependant critiquer les Descendantistes pour leurs idées. Dans cette situation, Zhang Cong eut également recours aux cas historiques de l'empereur Shun et de l'empereur Yu, mais pour aboutir à une conclusion inverse de celle des Descendantistes, ayant le principe de la piété filiale pour fondement - «bien traiter ses parents » - soit l'empereur doit honorer ses parents. Début novembre, Zhang Cong présenta à l'empereur un nouveau mémorandum et un article $D a$ Li Huo Wen.

(6) «Toutes les autres choses sont extérieures et les liens père-fils sont les liens éthiques les plus importants. " L'État et le trône sont extérieurs. Lorsque Gusou, père de l'empereur Shun commit un crime par homicide, Shun prit la fuite avec son père sur son dos sans se soucier du trône. L'empereur Shun a montré aux gens que sa relation père-fils avait plus d'importance que le trône et il avait mérité d'être hautement loué pour sa piété filiale et sa sagesse. En guise de réponse aux idées des grands secrétaires selon lesquelles l'empereur Shun, lorsqu'il succéda sur le trône à l'empereur Yao, ne tenta pas de donner de titre impérial à son père Gusou ni l'empereur Yu à son père Gun, d'où la conclusion que l'empereur Jiajing ne devrait pas non plus honorer son père, Zhang Cong souligna que, bien que Shun n'ait pas honoré son père Gusou, il ne prit pas l'empereur Yao pour père. L'empereur Yu, de même. Donc dans la présente affaire,

Argumentation et Analyse du Discours, 25 | 2020 
l'empereur Jiajing ne devait pas prendre l'empereur Xiaozong pour père, mais garder son père biologique le prince Xian de Xing comme père (Yang 1528, vol. 4 : 20a ; vol. 5, 1a-21b).

Une telle analyse nous permet de déduire que l'action discursive (6) observe les règles suivantes. Premièrement, la règle fonctionnelle de (6) est: dans un contexte où l'empereur Jiajing a menacé d'abdiquer et où les officiels étaient toujours encore en désaccord avec l'empereur, les ministres pouvaient choisir de proposer que l'empereur ne modifie pas sa parenté et maintienne sa décision d'honorer ses parents. Deuxièmement, la règle énonciative de (6) est : dans un contexte où l'empereur Jiajing a menacé d'abdiquer et où les officiels sont toujours encore en désaccord avec l'empereur, les sujets peuvent présenter un nouveau mémorandum et invoquer des événements historiques afin de réaliser la fonction ci-dessus.

\subsubsection{Concession des Descendantistes}

49 (7) «Le décret de l'impératrice Cishou est le suivant: Moi, votre empereur, je suis monté sur le trône. Mon père biologique, le prince Xian de Xing, devra être qualifié d'empereur Xingxian, ma mère biologique d'impératrice Xingxian et ma grand-mère d'impératrice Shao. C'est l'ordre donné par l'impératrice Cishou et je n'ose pas désobéir » (Yang $1528: 1 \mathrm{~b}:$ Gu 2015 :738).

Début novembre, comme la piété filiale de la progéniture envers ses parents, proposée par Zhang Cong, était irréfutable et que le prix de l'abdication de l'empereur était trop lourd, Yang Tinghe fit finalement une concession. Conformément à la fonction du cabinet intérieur de la dynastie Ming, le grand secrétaire avait la responsabilité de rédiger des édits. Ils rédigèrent alors, au nom de l'impératrice Cishou, un édit (cidessus) qui accorda respectivement au prince Xian de Xing et à la princesse Xian de Xing les titres d'empereur Xingxian et d'impératrice Xingxian. Jiajing ratifia alors l'édit. Il fut promulgué au nom de l'impératrice plutôt qu'en celui de la cour entière parce que cela exonérait les officiels de leurs responsabilités, leur permettait de sauver la face et montrait aux gens que cette décision n'avait pas été adoptée par toute la cour (Yang 1528:1b; Hu: 65). L'empereur Jiajing avait finalement réussi à conférer à ses parents l'honneur de titres impériaux, ce qui mettait fin à l'affaire. Le jour suivant, la mère de Jiajing arriva de Tongzhou et s'installa au palais.

51 Ce type de négociation permet de voir que l'action discursive (7) possède les règles argumentatives suivantes. Premièrement la règle fonctionnelle de (7) est: dans un contexte où l'empereur a menacé d'abdiquer et où Zhang Cong a présenté un nouveau mémorandum, le cabinet choisit d'accepter le fait que l'empereur honore ses parents conformément à l'injonction de piété filiale due par un fils à ses parents. Deuxièmement, la règle énonciative de (7) est: dans un contexte où l'empereur a menacé d'abdiquer et où Zhang Cong a présenté un nouveau mémorandum, le cabinet intérieur choisit de rédiger un décret au nom de l'impératrice Cishou afin de réaliser la fonction ci-dessus.

\subsection{Les règles argumentatives et le raisonnable}

Après avoir analysé les sept actions discursives de l'affaire du titre impérial, nous avons obtenu sept règles fonctionnelles et sept règles énonciatives. Nous allons montrer à présent que, dans le contexte culturel de la dynastie Ming, ces règles sont raisonnables. 
Nous verrons que parfois la même norme sociale pouvait valider des règles différentes et que la même règle pouvait être validée par différentes normes sociales. Aussi allonsnous, pour la commodité de l'analyse, regrouper ces règles en fonction des normes sociales.

Les sept règles fonctionnelles peuvent être divisées en deux groupes. Premièrement, les règles fonctionnelles de (1) et (4) respectent les normes sociales des lois du clan de la dynastie Ming. Comme indiqué initialement dans la section 2. 1., l'une des caractéristiques de la loi du clan est la primogéniture requérant que lorsqu'un grand progéniteur n'a pas d'héritier, un progéniteur mineur puisse prendre la succession de la grande primogéniture, le progéniteur mineur n'est cependant pas supposé conserver ses liens de descendance (par consanguinité) avec ses parents biologiques et peut se proclamer le descendant du grand progéniteur.

Les règles fonctionnelles de (2), (3), (5), (6), (7) suivent les normes sociales du principe de la piété filiale de la dynastie Ming. Comme indiqué initialement dans la section 2.2., la piété filiale requiert que "l'on traite bien ses parents", ce qui est à l'origine des coutumes et normes sociales intimant de garder de bonnes relations avec ses parents et de s'en occuper ; ceci constitue les valeurs fondamentales de la dynastie Ming.

Les sept règles énonciatives se répartissent en quatre groupes. Toutes les règles énonciatives de (1) à (7) sont conformes aux normes du système politique de la dynastie Ming. Comme indiqué initialement dans la section 2.3., les empereurs traitaient les affaires de l'État en invitant les officiels à débattre ou en promulguant des édits. Les règles énonciatives de (4) et (7) sont conformes à la fonction du cabinet intérieur. Celui-ci assume la responsabilité d'encourager les empereurs à faire de bonnes actions et à éviter les mauvaises. Lorsqu'un empereur a agi contrairement aux injonctions rituelles et aux lois ancestrales, le cabinet intérieur doit faire son maximum pour le corriger et même aller jusqu'à rejeter les édits des empereurs et les renvoyer (comme dans la règle énonciative (4)). Lorsque les empereurs étaient capables de bien justifier leurs actes, les officiels pouvaient faire des concessions. De plus, le cabinet intérieur est aussi responsable de la rédaction d'édits pour les empereurs (comme dans l'énonciation (7)). La règle énonciative (1) correspond à la responsabilité du Ministère des Rites. Lorsque les empereurs convoquent les officiels pour discuter d'affaires de l'État, les secteurs de l'administration publique sont censés discuter les affaires et transmettre ensuite les résultats de la discussion aux empereurs (Zhang et Lin 2003 : 205-206). Le ministère des Rites de la dynastie Ming était responsable des rituels d'État, des rituels sacrificiels etc. Lorsque l'empereur lui demandait de discuter des rites, c'était pour assumer ses responsabilités en matière de conseils à donner à l'empereur. En outre, les actes d'énonciation de (3), (5), (7) sont conformes au principe de piété filiale. Le fait que l'empereur ait promulgué un édit, invoqué le lien familial (règle énonciative (3), déclaré son intention d'abdiquer afin de s'occuper de sa mère (comme formulé dans (5) et promulgué l'édit au nom de l'impératrice Cishou afin d'appliquer l'ordre de sa mère (règle énonciative (7) - a démontré ce qu'était un fils dévoué.

Deuxièmement, les règles énonciatives de (3), (2), (4), (6) correspondent au style d'écriture des érudits Ming qui aimaient recourir à des événements historiques dans leurs écrits et dans leurs argumentations avec les autres. Ils pensaient qu'un emploi approprié d'événements historiques pouvait augmenter la force persuasive. Le recours aux exemples historiques à propos d'Ai, empereur de Han, Ying empereur de Song, de l'empereur Shun et de l'empereur Yu dans les règles énonciatives (1), (4) a contribué à 
clarifier les principes des lois du clan, alors que dans les règles énonciatives de (2) et (6) les mêmes événements historiques ont donné lieu à une interprétation opposée destinée à réfuter la thèse des opposants.

Troisièmement, la règle énonciative de (2) et (4) correspond aux styles des érudits Ming qui aimaient donner des citations confucéennes classiques dans leurs écrits et argumentaires. La culture confucéenne a été la culture dominante de la société chinoise traditionnelle. Dans ces deux règles, Li Ji (livre des rites) et Chun Qiu Gong Yang Zhuan sont des classiques faisant partie des 13 Classiques du Confucianisme, qui étaient très largement connus des érudits Ming et jouissaient alors d'une grande autorité.

Quatrièmement, les règles énonciatives de (1), (2), (4), (6), (7) répondent aux exigences des lois ancestrales de la dynastie Ming. Dans la catégorie Shen Guo Zheng de Huang Ming Zu Xun, il était stipulé que n'importe qui, indépendamment de son grade officiel dans l'administration ou de son activité en tant que roturier, pouvait signaler quelque chose à l'empereur. Toute déclaration, dès lors qu'elle était raisonnable, devait être acceptée et mise en œuvre (Zhu 1996 : 172). Comme indiqué dans 2.4., ce livre jouissait d'une grande autorité légale.

Après la justification des règles que nous avons déduites de l'étude de cas, les règles fonctionnelles et la règle du discours sont toutes deux promues au rang de normes sociales. L'argumentation, en tant que jeu de langage, est régulée dans sa progression par ces normes. L'argumentation en tant que jeu de langage fonctionne ainsi : motivé par le contexte initial du discours (1)), l'argumentateur ${ }_{1}$ formule la fonction du discours (1) qui a la règle fonctionnelle (1) pour fondement, puis produit le discours (1) ayant la règle discursive du discours pour fondement. Finalement, le discours (1) produit un effet contextuel sur l'autre argumentateur ${ }_{2}$ et son contexte d'origine. À ce moment, le premier mouvement est fini et le prochain commence muni de l'apport contextuel du discours (1): un nouveau contexte pour le discours (2) de l'argumentaeur ${ }_{2}$.Les mouvements suivants de ce jeu se poursuivent jusqu'à la fin de la même façon. Nous allons appliquer à présent ce jeu de langage de l'argumentation à notre cas, l'affaire du titre impérial, et décrire le processus entier comme suit (tableau 3):

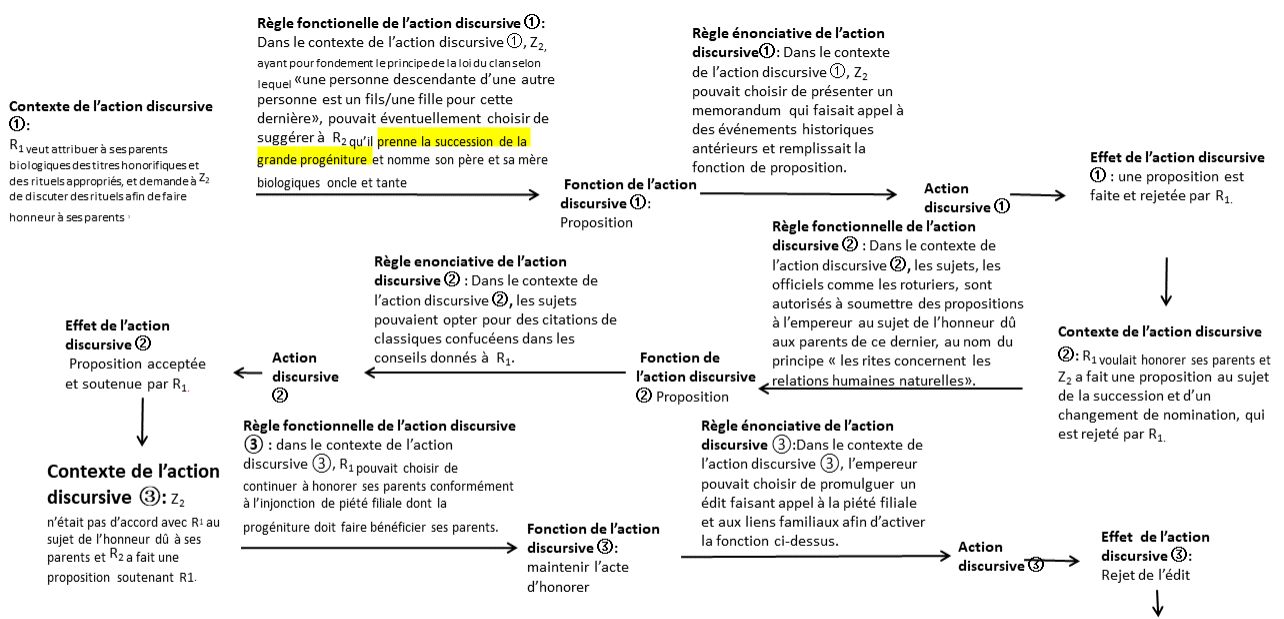




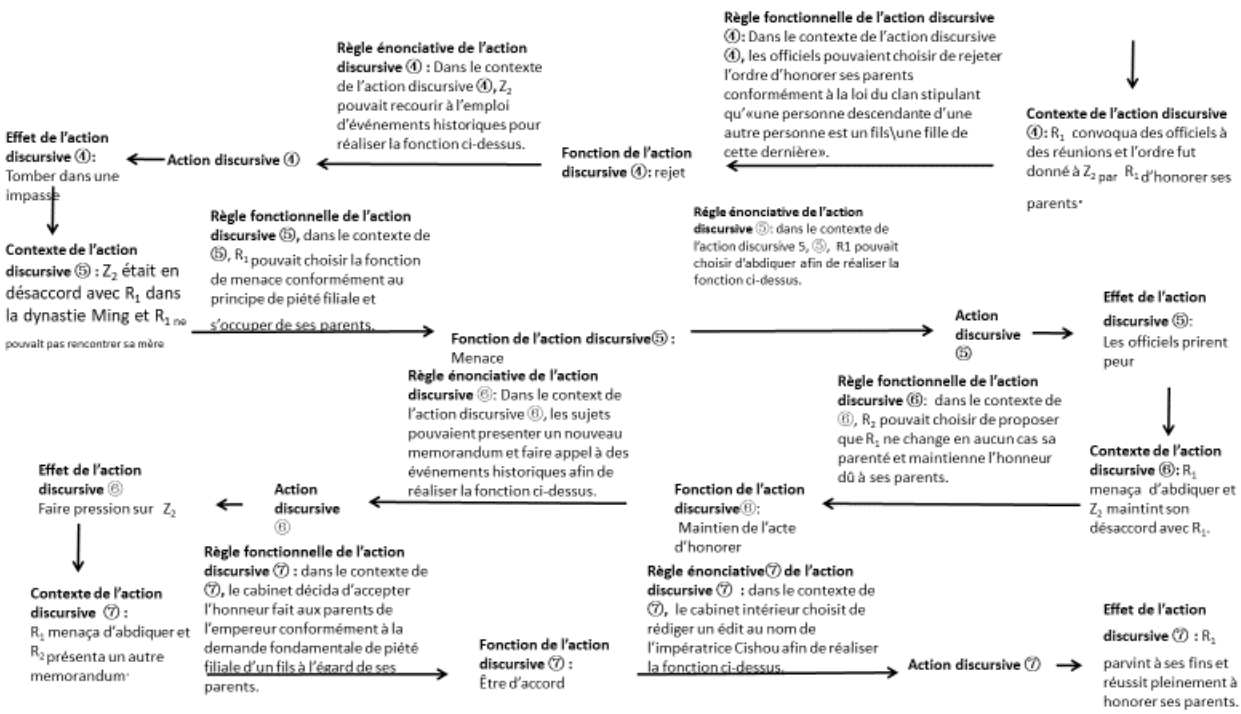

61 L'analyse des sections 4.1.-4.2. clarifie le fait que la question du titre impérial a démontré l'existence des traits distinctifs argumentatifs suivants: les deux camps partagent le système de rationalité de la Chine ancienne ainsi qu'un même contexte informationnel. Il y a cependant des différences de hiérarchisation. Chez les Descendantistes, on met davantage l'accent sur la référence à la tradition de la loi du clan, tandis que chez les Humanistes, on met davantage l'accent sur l'invocation de la tradition de la piété filiale, d'où des différences d'ordre traditionnel et de points de vue originels.

\subsection{Examen des règles}

Bien qu'ayant atteint son objectif dans cette affaire, l'empereur Jiajing était encore toujours insatisfait et il entreprit de tenter d'attribuer un titre honorifique plus élevé à ses parents, c'est-à-dire d'ajouter «Huang » et " Huanghou » (un titre plus spécifique) aux titres de ses parents et de les nommer Xingxian Huang et Xingxian Huanghou. C'est l'affaire Huang des sept affaires de la Controverse du Grand Rituel. Cette tentative de l'empereur fut la cible de fortes objections de la part de Yang Tinghe, le grand secrétaire, ainsi que de quelques autres officiels. Cela donna lieu à des débats houleux entre les deux camps. Jiajing dut suspendre temporairement sa tentative au terme de débats et de combats récurrents. Ce ne fut que deux ans plus tard, lorsque Yang démissionna de son poste, que l'empereur parvint à ses fins. Nous allons uniquement traiter ici de la première période de cette affaire.

Début décembre 1521, Jiajing ordonna tout d'abord aux officiels du ministère des rites de transmettre son édit, puis en remit plus tard une version écrite stipulant l'attribution à ses parents de titres plus honorifiques contenant Huang, soit Xingxian Huang et Xingxian Huanghou. Confronté à cette situation, Yang Tinghe et quelques autres officiels présentèrent leurs mémorandums; ils commencèrent par dire qu'ils comprenaient la décision de l'empereur, mais ils exprimèrent ensuite la crainte que cette tentative ne nuise à ses vertus. Ils considérèrent, en tant que grands secrétaires, que la tâche de contrôler l'empereur leur incombait et qu'ils n'avaient pas à s'attirer ses bonnes grâces. Dans leurs mémorandums, ils distinguèrent entre la loi du clan et les considérations personnelles. 
64 (8) Dès lors que votre majesté a succédé au règne de l'empereur Xiaozong et a pris Cishou pour mère, il serait logique qu'il y ait des différences entre son statut, son titre et ceux de votre mère biologique. Si votre majesté continue avec insistance à demander un titre plus honorifique pour vos parents biologiques, cela risquerait de provoquer un maelström de relations humaines et la transgression de la loi naturelle. C'est pourquoi nous sommes obligés de vous renvoyer votre édit et de revenir à notre précédente proposition (Zhang Juzheng et al., vol. $9: 4$; Yang Yiqing et al., vol. $7: 1 \mathrm{~b}-2 \mathrm{a}$ ).

Le discours (8) a appliqué deux règles. La fonction de la règle discursive(8) est premièrement la suivante : dans le contexte où un empereur Ming a promulgué un édit oral ou écrit afin de continuer à honorer ses parents, les officiels pouvaient choisir d'abroger l'ordre de l'empereur conformément à la loi du clan stipulant que « dans la loi du clan les parents de la lignée jouissent de plus grands honneurs que les parents biologiques ». C'est conforme aux normes de la tradition de la loi du clan (voir les règles fonctionnelles de (1) et (4)). Deuxièmement, la règle énonciative (8) est: dans le contexte où l'empereur Ming a promulgué un édit oral ou écrit afin de continuer à honorer ses parents, les officiels pouvaient abroger l'édit en présentant un mémorandum où une distinction était faite entre les parents selon la lignée de la loi du clan et les parents biologiques. Cette règle est conforme aux modalités d'organisation et aux arrangements institutionnels des grands secrétaires de la dynastie Ming (voir la règle énonciative de (4)).

Voyant que les grands secrétaires persistaient dans leur position, l'empereur Jiajing fit part sans détour de ses inquiétudes:

(9) Je sais que ce que vous avez dit est raisonnable et compréhensible. C'est juste que je ne peux pas me retenir d'aimer profondément mes parents biologiques et que je ne peux trouver de meilleur moyen d'exprimer ma profonde gratitude à leur égard pour m'avoir élevé. J'espère que vous ferez exactement ce que je vous ai demandé de faire et que vous ne direz jamais non (Zhang Juzheng et al., vol. 9:4 ; Yang Yiqing et al., vol. 7 : $1 \mathrm{~b}-2 \mathrm{a})$.

Le discours (9) comprend aussi deux règles. Premièrement, la règle fonctionnelle est : dans un contexte où les officiels se doivent de refuser l'ordre de l'empereur concernant l'attribution de titres honorifiques à ses parents, l'empereur pouvait l'imposer afin de réaliser l'objectif d'attribuer des titres honorifiques à ses parents en vertu de la norme de piété filiale stipulant que les enfants doivent s'occuper de leurs parents afin de leur témoigner leur gratitude (voir les règles fonctionnelles dans (2), (3), (5), (6), (7)). Deuxièmement, la règle énonciative de (9) est: dans le contexte où les officiels se doivent de rejeter l'ordre de l'empereur concernant l'attribution de titres honorifiques aux parents, l'empereur pouvait choisir de promulguer un édit faisant appel à un ethos humain afin de réaliser la fonction ci-dessus. Cette règle correspond au régime institutionnel de la dynastie Ming selon lequel l'empereur pouvait gouverner par promulgation d'édits (voir les règles énonciatives de (3), (6), (7)).

\section{Bilan}

Dans la Théorie de l'argumentation généralisée, l'argumentation est un mode d'interaction sociale que les membres d'une certaine communauté socio-culturelle mettent en œuvre afin de raisonner sur les choses. Elle considère les normes sociales de 
la communauté comme des règles argumentatives et se présente sous la forme d'une série d'actions discursives respectant ces règles. Les études argumentatives doivent avoir pour fondement le principe de localisation, interpréter l'action discursive et décrire les règles et structures argumentatives correspondantes du point de vue des participants de l'argumentation.

En ayant recours à la théorie ci-dessus, cet article a dégagé les règles et structures de l'argumentation concernant la question du titre impérial dans la Controverse du Grand Rituel de la dynastie Ming. La controverse consiste dans une série de questions à débattre au sujet de rites et du titre honorifique du père de l'empereur Jiajing. Il ne s'agit pas uniquement d'un combat idéologique entre le Descendantiste et l'Humaniste, mais aussi d'un combat pour le pouvoir entre les officiels représentés par les grands secrétaires et le jeune empereur récemment monté sur le trône.

Cela a manifestement démontré que les normes sociales, les stratégies et les figures de rhétorique ont été utilisées dans la procédure argumentative qui s'est déroulée entre l'empereur qui avait le dessous et les officiels qui avaient le dessus. Durant la procédure de l'argumentation, le proposant et l'opposant ont tous deux suivi le système politique de la Chine ancienne et ses traditions culturelles; ils ont mis les actions discursives en œuvre dans les limites des restrictions institutionnelles. En défendant leurs revendications, les deux camps sont également capables de se servir des théories des classiques anciens et des événements historiques. Mais les règles argumentatives qu'ils appliquent proviennent de normes sociales ayant la tradition de la piété filiale pour fondement, et de normes ancrées dans la tradition de la loi du clan. Enfin, grâce à l'interprétation socio-culturelle, l'argumentation concernant la seconde question a été décrite, évaluée et interprétée dans le cadre de la Théorie de l'argumentation généralisée.

\section{BIBLIOGRAPHY}

\section{Livres}

(Ming dynasty) (明) Zhu Yuan-zhang (朱元璋). (1996). Huang Ming Zu Xun (《皇明祖训》). Si Ku Quan Shu Cun Mu Cong Shu (《四库全书存目丛书》) History section(史部) 264th Book (第264册 ). Jinan: Qilu Press (济南 : 齐鲁书社)

(Ming dynasty) (明) Yang Ting-he et al. (杨廷和等). (1962-1968). Ming Wu Zong Shi Lu (《明武宗 实录》). Taipei: Institute of History and Philology, Academia Sinica (台湾“中央”研究院历史语言 研究所)

(Ming dynasty) (明) Zhang Ju-zheng et al. (张居正等). (1962-1968) Ming Shi Zong Shi Lu (《明世宗 实录》). Taipei: Institute of History and Philology, Academia Sinica (台湾“中央”研究院历史语言 研究所)

(Ming dynasty) (明) Yang, Yi-qing et al. (杨一清等). (1528). Ming Lun Da Dian (《明伦大典》). Jiajing 8th year, Huguang Edition (嘉靖八年.湖广刻本) 
(Qing dynasty) (清) Gu, Ying-tai (谷应泰). (1974). Origin of records of History of Ming dynasty(《明史 纪事本末》). Beijing: Zhonghua Book Company (北京 : 中华书局)

(Qing dynasty) (清) Zhang, Ting-yu (张廷玉). (1974). History of Ming dynasty. Beijing: Zhonghua Book Company (北京 : 中华书局)

Hu, Fan (胡凡). (2004). Bibliography of Jiajing emperor (《嘉靖传》). Beijing: People’s Publishing House (北京：人民出版社)

Liu Guang-ming (刘广明). (2011). Clan law of China - shaping, improving and motivation of social form of clan law (《宗法中国一一中国宗法社会形态的定型、完型和发展动力》). Nanjing: Nanjing University Press (南京 : 南京大学出版社)

Qu Tong-zu (罌同祖). (2017). Chinese feudal society (《中国封建社会》). Bejing: Commercial Press (北京：商务印书馆).

Wang You-tian (王天有). (2014). A study of state institutes of Ming dynasty (《明代国家机构研究》). Beijing: Palace Museum Publishing house (北京 : 故宫出版社)

Van Maanen, John. (2011). Tales of the Field: On Writing Ethnography (2nd edition). Chicago IL: University of Chicago Press

Zhang, Xian-qing (张显清) and Lin Jin-shu (林金树). (2003). ed. Political history of Ming dynasty( 《明代政治史》). Guilin: Guangxi Normal University (桂林：广西师范大学出版社)

Tian Shu (田㰾). (2013). Zhengde 16th year - the Great Ritual Controversy and Jia Long Wan reforms ( 《正德十六年-一“大礼议与嘉隆万改革》). Beijing: People's publishing house (北京: 人民出版社 )

Watson, Burton (2007). The Analects of Confucius. Trans. New York: Columbia University Press Zhao, Ke-sheng (赵克生). (2012). State rituals and social life of Ming dynasty（《明代国家礼制与社会 生活》). Beijing: Zhonghua Book Company (北京：中华书局)

\section{Articles}

Ju, Shi-er (鞠实儿). 2020.Generalized Argumentation Theoryand Methods (广义论证的理论与 方法). Studies in Logic (《逻辑学研究》) 13-1: 1-27

Ju, Shi-er (鞠实儿). 2012. Cultural relativism and cultural conflict solution. Invited Report in Department of Sociology, University of Wisconsin-Madison

Ju, Shi-er. 2010. The Cultural Relativity of Logic: From the Viewpoint of Ethnography and Historiography. Social Sciences in China 31 (4): 73-89

Ju, Shi-er (鞢实儿) and He, Yang (何杨). 2014. An inquiry into ancient Chinese logic from the viewpoint of the General Argumentation Theory - In the example of Political argumentation by reciting poems in the Spring and Autumn Period. Philosophical Researches (《哲学研究》)

(1): $102-110$

Li, Wen-zhi (李文治). (1988).Forms of clan law and their roles in the grass-root governments (明 代宗族制的体现形式及其基层政权作用-一论封建所有制是宗法宗族制发展变化的最终根源). Researches in Chinese economic history (《中国经济史研究》)

Wu, Zhi-he (吴智和). (1991). Interpretation and functional analysis of Ming ancestral laws (明代 祖制释意与功能试论). Collected Papers of History Studies (《史学集刊》) 
Xiao Qun-zhong (肖群忠). (2000). Filial piety and Chinese people's nature (孝与中国国民性). Philosophical Researches (《哲学研究》)

You Shu-jun (尤淑君). (2006). Ming Fen Li Zhi Yu Huang Quan Chong Su: Da Li Yi Yu Jia Jing Zheng Zhi Wen Hua (《名分礼秩与皇权重塑 : 大礼议与嘉靖政治文化》). Paipei: National Chengchi University (台北: 政治大学历史系

Zhang Jian (张践). (2000). Origin and development of Confucian view of filial piety (儒家孝道观的 形成于演变). History of Chinese philosophy (《中国哲学史》)

\section{NOTES}

1. Nous sommes reconnaissants à tous ceux qui nous ont aidé à améliorer cet article, et remercions le Fonds national de science sociale de Chine pour son soutien (18ZDA033).

2. Il est communément admis qu'il existe deux sortes de règles argumentatives. La première est généralement définie dans les théories occidentales de l'argumentation où le concept de type d'argument est plutôt un concept formel. L'autre type est celui des normes sociales telles que définies dans la Théorie généralisée de l'argumentation (Generalized Argumentation Theory).

3. Dans les dynasties féodales chinoises, la polygamie était légale. Le roi ou l'empereur a plusieurs épouses, à la résidence royale, et parmi elles, la reine ou l'impératrice est habituellement la consort principale (parmi les épouses), dont les fils aînés peuvent légalement monter sur le trône impérial.

4. Zhu Yuanzhang, Tai Zu empereur, est né en 1328 et a régné à partir de 1368 ; il est mort en 1398. Toute référence à un autre empereur sera conforme à ce format.

5. L'évolution du cabinet intérieur est compliquée. Ses responsabilités ne peuvent pas être uniquement considérées comme celles du conseiller. - L'enjeu de cet article n'est pas l'investigation de la fonctionnalité de ce cabinet intérieur. Donc nous n'allons pas la discuter en détails.

6. L'ascension est un rituel accompli quand un empereur est couronné instance suprême du pouvoir de direction du pays.

7. Il existe une solution supplémentaire proposant que le nouvel empereur soit obligé de succéder par descendance et de devenir un fils de son cousin (Yang $1528: 10-11$ ). Cette solution ne retint pas particulièrement l'attention. Aussi allons-nous l'ignorer dans cet article.

8. Le problème des rites fut résolu en 1538 lorsqu'un temple fut érigé pour lui.

9. La date finale de la controverse est encore controversée. Quelques chercheurs pensent que la phase finale devrait probablement être fin octobre 1524, parce qu'à cette époque la question de la paternité était réglée. D'autres pensent qu'il devrait s'agir de début juillet 1528, lorsque Ming Lun Da Dian, le document officiel qui mit un terme à la controverse, fut publié (You, 2006 :150). Il y en a encore d'autres qui situent la fin en 1538, année où le temple sacrificiel familial dédié au père de l'empereur Jiajing fut inauguré (Zhang 2003 : 9).

10. Dans l'affaire du titre impérial, les grands secrétaires du Cabinet Intérieur incluent Yang Tinghe, Jiang Mian et Mao Ji.

11. Le Ministère des Rites avait invité des officiels à discuter des rites et du titre et, après les discussions, le ministère présenta des mémorandums à l'empereur dirigés par Mao Ji, l'un des trois grands secrétaires. 


\section{ABSTRACTS}

By using the Generalized Argumentation Theory, this paper tries to reveal the rules and structures of the argumentation about the imperial title issue in the Great Ritual Controversy. Our analysis shows that both the protagonist and the antagonist follow ancient Chinese political system and cultural traditions, and appeal to ancient classics and historical events in order to defend their claims. With these rules, the argumentation about the issue is demonstrated. Finally, this paper shows that: 1) Generalized Argumentation Theory and its methods are tolerant of cultural pluralism (including mainstream culture);2) it is feasible to describe and evaluate arguments through socio-cultural interpretation.

Le recours à la "Théorie généralisée de l'argumentation » cherche à dégager les règles et les structures de l'argumentation dans la controverse dite du « Grand Rituel » autour de la titulature impériale. Notre analyse montre que le Proposant et l'opposant adhèrent tous deux à l'ancien système politique et aux traditions culturelles anciennes et se réfèrent aux classiques et à des événements historiques du passé afin de défendre leurs thèses. L'argumentation concernant cette question est démontrée via ces règles. En résumé, cet article montre que : 1) la Théorie généralisée de l'argumentation et sa méthode acceptent le pluralisme culturel (culture dominante incluse) ; 2) il est possible de décrire et d'évaluer des arguments en recourant à une interprétation socio-culturelle.

\section{INDEX}

Mots-clés: argumentation généralisée, argumentation politique dans la dynastie Ming, interprétation socio-culturelle, logique de la Chine ancienne

Keywords: ancient Chinese logic, generalized argumentation, political argumentation in Ming dynasty, socio-cultural interpretation

\section{AUTHORS}

\section{JU SHI'ER}

Institute of Logic, Cognition and Philosophy Department, Sun Yat-sen University

\section{LIU WEN}

Institute of Logic, Cognition and Philosophy Department, Sun Yat-sen University

\section{CHEN ZHIXI}

Institute of Logic, Cognition and Philosophy Department, Sun Yat-sen University 